\title{
Positron Emission Tomography/Computed Tomography in the Staging of Colon Cancer
}

\author{
Jae Hyung Lee, Min Ro Lee \\ Research Institute of Clinical Medicine, Chonbuk National University Medical School, Jeonju, Korea
}

Purpose: Accurate preoperative staging of colon cancer is essential for providing the optimal treatment strategy and evaluating the expected prognosis. The aim of this study is to assess the value of positron emission tomography/computed tomography (PET/CT) over conventional studies in the staging of colon cancer.

Methods: A total of 266 colon cancer patients diagnosed between January 2008 and December 2010 were assessed with both PET/CT and conventional studies. Discordance with PET/CT and conventional studies were evaluated, and changes in the management strategy were assessed for each stage. Discordant findings were verified by using intraoperative examination, pathology reports, and follow-up imaging studies.

Results: Multidetector computed tomography (MDCT) and PET/CT showed similar accuracy in detecting lymph node metastasis in patients with clinical stage III $(36.2 \%$ vs. $42 \%, \mathrm{P}=0.822)$ and stage IV $(60.3 \%$ vs. $63.5 \%, \mathrm{P}=0.509)$ disease. $\mathrm{PET} / \mathrm{CT}$ led to a change in management strategy for 1 of 40 patients (2.5\%) with clinical stage I, 0 of 25 patients (0\%) with stage II, 9 of 138 patients (6.5\%) with stage III, and 8 of 63 patients (12.7\%) with stage IV disease.

Conclusion: PET/CT changed the management plan in $6.5 \%$ of patients with clinical stage III and $12.7 \%$ of patients with clinical stage IV colon cancer. Our findings suggest that PET/CT may be considered as a routine staging tool for clinical stage III and IV colon cancers.

Keywords: Positron emission tomography/computed tomography; Colon neoplasms; Staging

\section{INTRODUCTION}

Accurate preoperative staging of colon cancer is essential for assessing prognosis and determining appropriate treatment. Preoperative evaluation and staging should focus on techniques that might preclude surgery entirely, lead to a change in the preoperative or intraoperative surgical plan, or indicate the need for preoperative neoadjuvant therapy [1].

Abdomino-pelvic computed tomography $(\mathrm{CT})$ and chest $\mathrm{CT}$ are

Received: August 11, 2013 - Accepted: October 12, 2013

Correspondence to: Min Ro Lee, M.D.

Research Institute of Clinical Medicine, Chonbuk National University

Medical School, 20 Geonji-ro, Deokjin-gu, Jeonju 561-712, Korea

Tel: +82-63-250-1570, FAX: +82-63-271-6197

E-mail:gsminro@chonbuk.ac.kr

(c) 2014 The Korean Society of Coloproctology

This is an open-access article distributed under the terms of the Creative Commons Attribution NonCommercial License (http://creativecommons.org/licenses/by-nc/3.0) which permits unrestricted noncommercial use, distribution, and reproduction in any medium, provided the original work is properly cited. standard imaging modalities for the staging of colon cancer. Despite technical improvements, such as multidetector CT (MDCT), an ideal preoperative staging has not yet been obtained [2]. Ahmetoglu et al. [3] reported an overall accuracy of MDCT of $86 \%$ in T staging and $84 \%$ in $\mathrm{N}$ staging. A meta-analysis demonstrated the sensitivities of CT and positron emission tomography (PET) to be $83.6 \%$ and $94.1 \%$, respectively, for detecting hepatic metastasis from colorectal cancer (CRC) [4].

Currently, PET/CT is recommended only for the assessment of suspected recurrence of CRC and in the preoperative staging prior to a metastasectomy [2]. Reports on the role of PET/CT in the routine staging of colon cancer are scarce and conflicting $[2,5,6]$. Colon cancers differ from rectal cancers in their pathways before surgery, and the algorithms for the treatments of rectal cancer are more complex. For this reason, our study included only colon cancer. The aim of this study is to assess the value of PET/CT over conventional imaging studies (abdomino-pelvic CT and chest CT) in the preoperative staging of colon cancer. 


\section{METHODS}

We retrospectively analyzed the medical records of 266 colon cancer patients treated at Chonbuk National University Medical School, Jeonju, Korea from January 2008 to December 2010. All 266 patients underwent both PET/CT and conventional imaging studies preoperatively. We excluded patients who did not undergo preoperative CT or PET/CT, who underwent conventional image studies or PET/CT at another institution, or who underwent local excision, a palliative colostomy or ileostomy formation. As a result, a total of 266 colon cancer patients were reviewed.

The conventional staging MDCT scans and PET/CT were reported separately by a dedicated specialist radiologist and specialist nuclear medicine physician. CT examinations were performed on a 16-MDCT scanner (Sensation 16, Siemens HealthCare, Erlangen, Germany). The images were acquired with a $1.5 \times 16$ beam collimation. The other scanning parameters were as follows: $160 \mathrm{mAs}, 120 \mathrm{kVp}, 1.5-\mathrm{mm}$ detector collimation, 24-mm per rotation table speed, and a 0.5 -second gantry rotation time. A reconstruction slice thickness of $3.0 \mathrm{~mm}$ and a reconstruction interval of $3.0 \mathrm{~mm}$ were also established.

The PET/CT examinations were performed using a standardized protocol on a two-detector-row PET-CT scanner (Siemens Medical Solution, Hoffman Estates, IL, USA). Patients were asked to fast for at least 6 hours before examination to ensure that the blood glucose level was below $150 \mathrm{mg} / \mathrm{dL}$. At 1 hour after fluorodeoxyglucose administration, a low-dose CT scan was performed for PET-attenuation correction, covering the neck, thorax, abdomen, and pelvis ( $80 \mathrm{~mA}, 120 \mathrm{kV}, 3.75-\mathrm{mm}$ slice thickness).

Discordance with PET/CT and conventional imaging studies were evaluated, and changes in the management strategy were assessed at each stage. The T, N, and M staging was based on American Joint Committee on Cancer 6th guidelines for colon cancer. We obtained sensitivities, specificities, positive predictive values
(PPVs), negative predictive values (NPVs), and accuracies between MDCT and PET/CT for lymph node metastasis at stage III and IV. We reviewed the medical records of all patients, and discordant findings were verified by using intraoperative findings or examination, pathology reports, and follow-up imaging studies after surgery.

For lymph node metastasis, the sensitivity, specificity, PPV, NPV, and accuracies of MDCT and PET/CT were calculated. The differences in the accuracies for lymph node metastasis between PET/ CT and MDCT were assessed using IBM SPSS ver. 19.0 (IBM Co., Armonk, NY, USA). Comparisons were assessed by using the chisquare test. A P-value $<0.05$ was considered statistically significant.

\section{RESULTS}

The 266 patients enrolled in this study had a mean age of 63.7 years (range, $35-87$ years); 112 patients $(42.1 \%)$ were female, and 154 patients $(57.9 \%)$ were male. In the patients with clinical stage III cancer $(\mathrm{n}=138)$, for regional lymph node metastasis, MDCT had a sensitivity of $100 \%$, a specificity of $0 \%$, a PPV of $36.2 \%$, an accuracy of $36.2 \%$. The NPV could not be calculated. In comparison, PET/CT had a sensitivity of $88 \%$, a specificity of $15.9 \%$, a PPV of $37.3 \%$, a NPV of $70 \%$, and an accuracy of $42 \%$. The difference in accuracy was not statistically significant $(\mathrm{P}=0.822)$.

For regional lymph node metastasis in the group with clinical stage IV cancer $(n=63)$, MDCT had a sensitivity of $97.4 \%$, a specificity of $4 \%$, a PPV of $60.7 \%$, a NPV of $50 \%$, and an accuracy of $60.3 \%$. In comparison, PET/CT had a sensitivity of $97.4 \%$, a specificity of $12 \%$, a PPV of $62.7 \%$, a NPV of $75 \%$, and an accuracy of $63.5 \%$. The difference in accuracy was not statistically significant $(\mathrm{P}=0.509)$ (Table 1$)$.

Eighteen patients (6.8\%) underwent potential changes in management plan as a result of the PET/CT findings. Potential upstaging was considered in four patients. PET/CT revealed findings for

Table 1. Sensitivity, specificity, PPV, NPV, and accuracy for lymph node metastasis in stage III and IV colon cancer patients

\begin{tabular}{lccccccc}
\hline Stage & Modality & Sensitivity (\%) & Specificity (\%) & PPV (\%) & NPV (\%) & Accuracy (\%) & P-value \\
\hline III & MDCT & 100 & 0 & 36.2 & C & 36.2 & 0.822 \\
IV & PET/CT & 88.0 & 15.9 & 37.3 & 70.0 & 42.0 \\
& MDCT & 97.4 & 4.0 & 60.7 & 50.0 & 60.3 & 0.509 \\
\hline
\end{tabular}

PPV, positive predictive value; NPV, negative predictive value; MDCT, multidetector computed tomography; C, could not calculated; PET/CT, positron emission tomography/ computed tomography.

Table 2. Potential upstage after PET/CT

\begin{tabular}{lllcc}
\hline No. of patients & \multicolumn{1}{c}{ Cl findings } & PET/CT finding & Stage change & Verification \\
\hline 3 & No liver metastasis & Liver metastasis & $\||| \rightarrow \mid V$ & Histology; liver metastasis \\
1 & No peritoneal seeding & Peritoneal seeding & ||$|\rightarrow| V$ & Intraoperative findings; peritoneal seeding \\
\hline
\end{tabular}

PET/CT, positron emission tomography/computed tomography; Cl, conventional image. 
Table 3. Potential downstage after PET/CT

\begin{tabular}{|c|c|c|c|c|}
\hline No. of patients & Cl finding & PET/CT finding & Stage change & Verification \\
\hline 4 & Lung metastasis & No lung metastasis & $\|\mathrm{V} \rightarrow \mid\|$ & Follow-up CT; no change \\
\hline 1 & Lung metastasis & No lung metastasis another ascending colon cancer & $\mid \mathrm{V} \rightarrow \|$ & Follow-up CT; no change; histology \\
\hline 1 & $\begin{array}{l}\text { Distant lymph nodes, } \\
\text { liver metastasis }\end{array}$ & No metastasis & $\mathrm{IV} \rightarrow \mathrm{II}$ & $\begin{array}{l}\text { Follow-up CT; no change; liver MRI; } \\
\text { hemangioma }\end{array}$ \\
\hline 1 & Liver metastasis & Eosinophilic abscess or liver metastasis & $I V \rightarrow I \| I$ or IV & Histology; eosinophilic abscess \\
\hline 1 & Metastatic tumor seeding & No seeding & $|V \rightarrow| \| \mid$ & Intraoperative findings; no seeding \\
\hline 1 & No rectal polyp & Rectal polyp & $\|\rightarrow\|$ & $\begin{array}{l}\text { Intraoperative findings; rectal } \\
\text { polypectomy }\end{array}$ \\
\hline
\end{tabular}

PET/CT, positron emission tomography/computed tomography; Cl, conventional image; CT, computed tomography.

Table 4. Proportion of management changes after PET/CT

\begin{tabular}{|c|c|c|c|c|c|c|c|}
\hline \multicolumn{2}{|c|}{ Conventional stage } & \multicolumn{4}{|c|}{ Post-PET/CT stage (n) } & \multicolumn{2}{|c|}{ Management change } \\
\hline Stage & No. of patients & Stage I & Stage II & Stage III & Stage IV & No. of patients & $\%$ \\
\hline I & 40 & 29 & 0 & 11 & 0 & 1 & 2.5 \\
\hline$\|$ & 25 & 0 & 13 & 10 & 2 & 0 & 0 \\
\hline III & 138 & 0 & 20 & 106 & 12 & 9 & 6.5 \\
\hline IV & 63 & 0 & 4 & 16 & 43 & 8 & 12.7 \\
\hline
\end{tabular}

PET/CT, positron emission tomography/computed tomography.

liver metastasis in three patients and peritoneal seeding in one patient; these had not been shown by conventional studies. According to the PET/CT findings, combined liver resections were performed on three patients, and the patients were confirmed with liver metastasis by using pathologic examinations. One patient who was suspected of having peritoneal seeding on PET/CT had this suspicion confirmed by intraoperative findings (Table 2). Potential downstaging was indicated in nine patients. Five patients were suspected of having lung metastasis on conventional staging while the lesions were suspected as being benign on PET/CT. Follow-up image studies revealed benign lung nodules. In addition to stage migration, the PET/CT suggested another colonic cancer lesion in one patient, on whom a total colectomy was performed.

One patient demonstrated a distant lymph node metastasis and liver metastasis on conventional studies. However, PET/CT indicated a benign lesion for a distant lymph node, and the lesion was identified as benign in a series of follow-up studies. For the liver lesion, additional magnetic resonance imaging (MRI) was performed and demonstrated a hemangioma. One patient with a suspicious liver lesion on conventional studies showed liver metastasis or an eosinophilic abscess on PET/CT. Pathologic findings were compatible with an eosinophilic abscess. One patient demonstrated mesenteric tumor seeding on MDCT while PET/CT findings were negative. Intraoperative findings revealed no mesenteric tumor seeding. Another patient with a suspicious stage III on conventional staging was reconsidered as stage II after PET/CT. In addition, a rectal polyp was found on PET/CT, and the patient underwent an intraoperative polypectomy (Table 3).
As well as stage migration, the PET/CT suggested additional pathologic lesions in five patients, resulting in changed management strategies. Another colonic lesion was found in two patients. One underwent a polypectomy after surgery, and the other had a changed operative plan. Additional findings on PET/CT included cases of thyroid cancer, a pituitary macroadenoma, and an adenocarcinoma on the antrum of the stomach. The 18 patients who underwent changes in management plan included 1 of $40(2.5 \%)$ stage I, 0 of $25(0 \%)$ stage II, 9 of 138 (6.5\%) stage III, and 8 of 63 (12.7\%) stage IV (Table 4).

\section{DISCUSSION}

This study demonstrated that the addition of PET/CT to conventional imaging studies changed the management plan in $6.5 \%$ of patients with clinical stage III colon cancer and $12.7 \%$ of patients with clinical stage IV colon cancer. Only a few studies have investigated the role of PET/CT (including PET) in the staging of colon cancer [2, 5-10], and the results vary. One study reported that routine use of PET/CT for preoperative staging did not impact disease management for $96.8 \%$ of patients, and that only $3.2 \%$ of patients had a change in surgical management. The researchers concluded that PET/CT should not be routinely used for primary staging of CRC [2]. On the other hand, another study reported that PET findings modified the scope of surgery in $11.54 \%$ of the patients and revealed an unknown disease in $19.2 \%$. These authors claimed that, when compared with conventional techniques, PET appeared to be useful in presurgical staging of CRC [6]. However, most of 
these studies included small numbers of CRC patients. To our knowledge, our study is the largest one published.

Our results showed that the preoperative staging of clinical stage III and IV colon cancer was changed in $6.0 \%$ to upstaging and in $19.9 \%$ to downstaging by addition of PET/CT as a staging modality. Upstaging was mainly due to detection of hepatic metastases. Given the known benefits of a hepatic metastasectomy, PET/CT should play an important role in the management of advanced colon cancer by detecting hepatic metastases. A meta-analysis demonstrated the sensitivities of CT, MRI, and PET for detecting hepatic metastases from CRC to be $83.6 \%, 88.2 \%$, and $94.1 \%$, respectively [4]. Modern MRI is superior to CT for detecting lesions that measure less than $1 \mathrm{~cm} \mathrm{[4].} \mathrm{PET} \mathrm{is} \mathrm{accurate} \mathrm{for} \mathrm{identification} \mathrm{of}$ hepatic metastases greater than $1 \mathrm{~cm}$ in diameter, but it is limited in its ability to demonstrate lesions smaller than $1 \mathrm{~cm}$ [11-13]. PET/CT may be particularly useful in patients with several hypodense or hypoenhancing liver lesions that are not clearly characterized by $\mathrm{CT}$ alone and in patients in whom standard CT fails to detect metastases in the setting of a rising serum CEA level [14].

Five patients who were clinical stage IV due to lung metastasis after conventional staging were considered as stage III after PET/ $\mathrm{CT}$. The chest CT indicated metastatic lung lesions; however, on $\mathrm{PET} / \mathrm{CT}$, the lesions were suspected to be benign or indeterminate lesions. No studies have yet compared chest CT versus PET/CT findings for metastatic lung lesions. Further studies should be performed.

In the aspect of cost effectiveness, one study reported that the use of PET/CT for preoperative evaluation in primary CRC was not economic [15]. However, only two small studies evaluated the use of PET/CT in primary CRC, and evidence supporting its routine use was insufficient. For economic evaluation, more studies are still needed.

As mentioned earlier, a few studies have investigated the benefits of PET/CT as a staging modality for CRC, and the results are controversial. In our study, PET/CT found incidental lesions in five patients. We did not focus on incidental findings of PET/CT that did not change the management plans; for example, some patients revealed incidental thyroid nodules after PET/CT. Those findings did not influence the management plans, so we did not concentrate on them. Although we only focused on findings after PET/CT that changed the management plans, we found that 18 of 266 patients (6.8\%) had changed management plans after PET/CT. In patients with conventional stage III and IV, 9 of 138 patients (6.5\%) and 8 of 63 patients (12.7\%) had changed methods of management. The results of our study, as the largest one yet published, suggest that PET/ CT might be considered as a valuable staging modality, especially in clinical stage III and IV colon cancer patients.

In conclusion, PET/CT changed the management plans in $6.5 \%$ of patients with clinical stage III colon cancer and $12.7 \%$ of patients with clinical stage IV colon cancer. Routine PET/CT may not be essential for all patients, but patients with clinical stage III and IV colon cancer should be considered for additional preoperative
$\mathrm{PET} / \mathrm{CT}$ after conventional studies. Additional research is required to select the group of patients that might benefit from PET/CT as an initial staging modality.

\section{CONFLICT OF INTEREST}

No potential conflict of interest relevant to this article was reported.

\section{REFERENCES}

1. Cohen AM. Preoperative evaluation of patients with primary colorectal cancer. Cancer 1992;70(5 Suppl):1328-32.

2. Cipe G, Ergul N, Hasbahceci M, Firat D, Bozkurt S, Memmi N, et al. Routine use of positron-emission tomography/computed tomography for staging of primary colorectal cancer: does it affect clinical management? World J Surg Oncol 2013;11:49.

3. Ahmetoglu A, Cansu A, Baki D, Kul S, Cobanoglu U, Alhan E, et al. MDCT with multiplanar reconstruction in the preoperative local staging of rectal tumor. Abdom Imaging 2011;36:31-7.

4. Niekel MC, Bipat S, Stoker J. Diagnostic imaging of colorectal liver metastases with CT, MR imaging, FDG PET, and/or FDG PET/ CT: a meta-analysis of prospective studies including patients who have not previously undergone treatment. Radiology 2010;257: 674-84.

5. Park IJ, Kim HC, Yu CS, Ryu MH, Chang HM, Kim JH, et al. Efficacy of PET/CT in the accurate evaluation of primary colorectal carcinoma. Eur J Surg Oncol 2006;32:941-7.

6. Llamas-Elvira JM, Rodriguez-Fernandez A, Gutierrez-Sainz J, Gomez-Rio M, Bellon-Guardia M, Ramos-Font C, et al. Fluorine-18 fluorodeoxyglucose PET in the preoperative staging of colorectal cancer. Eur J Nucl Med Mol Imaging 2007;34:859-67.

7. Akiyoshi T, Oya M, Fujimoto Y, Kuroyanagi H, Ueno M, Yamaguchi T, et al. Comparison of preoperative whole-body positron emission tomography with MDCT in patients with primary colorectal cancer. Colorectal Dis 2009;11:464-9.

8. Furukawa H, Ikuma H, Seki A, Yokoe K, Yuen S, Aramaki T, et al. Positron emission tomography scanning is not superior to whole body multidetector helical computed tomography in the preoperative staging of colorectal cancer. Gut 2006;55:1007-11.

9. Tsunoda Y, Ito M, Fujii H, Kuwano H, Saito N. Preoperative diagnosis of lymph node metastases of colorectal cancer by FDG-PET/ CT. Jpn J Clin Oncol 2008;38:347-53.

10. Tateishi U, Maeda T, Morimoto T, Miyake M, Arai Y, Kim EE. Non-enhanced CT versus contrast-enhanced CT in integrated PET/CT studies for nodal staging of rectal cancer. Eur J Nucl Med Mol Imaging 2007;34:1627-34.

11. Fong Y, Saldinger PF, Akhurst T, Macapinlac H, Yeung H, Finn $\mathrm{RD}$, et al. Utility of 18F-FDG positron emission tomography scanning on selection of patients for resection of hepatic colorectal metastases. Am J Surg 1999;178:282-7.

12. Rohren EM, Paulson EK, Hagge R, Wong TZ, Killius J, Clavien 
PA, et al. The role of F-18 FDG positron emission tomography in preoperative assessment of the liver in patients being considered for curative resection of hepatic metastases from colorectal cancer. Clin Nucl Med 2002;27:550-5.

13. Sahani DV, Kalva SP, Fischman AJ, Kadavigere R, Blake M, Hahn $\mathrm{PF}$, et al. Detection of liver metastases from adenocarcinoma of the colon and pancreas: comparison of mangafodipir trisodiumenhanced liver MRI and whole-body FDG PET. AJR Am J Roentgenol 2005;185:239-46.
14. O'Connor OJ, McDermott S, Slattery J, Sahani D, Blake MA. The Use of PET-CT in the Assessment of Patients with Colorectal Carcinoma. Int J Surg Oncol 2011;2011:846512.

15. Brush J, Boyd K, Chappell F, Crawford F, Dozier M, Fenwick E, et al. The value of FDG positron emission tomography/computerised tomography (PET/CT) in pre-operative staging of colorectal cancer: a systematic review and economic evaluation. Health Technol Assess 2011;15:1-192. 